Acta Crystallographica Section A

Foundations of Crystallography

ISSN 0108-7673

Received 4 July 2012

Accepted 8 November 2012

\section{Lawrence Bragg, microdiffraction and X-ray lasers}

\author{
J. C. H. Spence
}

Department of Physics, Arizona State University, Tempe, AZ 85282, USA. Correspondence e-mail:
spence@asu.edu

We trace the historical development of W. L. Bragg's 'law' and the key experimental observation which made it possible using polychromatic radiation at a time when neither X-ray wavelengths nor cell constants were known. This led, through his phasing and solving large mineral structures (without use of a computer), to work on metals, proteins, bubble rafts and his X-ray microscope. The relationship of this to early X-ray microdiffraction is outlined, followed by a brief review of electron microdiffraction methods, where electron-probe sizes smaller than one unit cell can be formed with an interesting 'failure' of Bragg's law. We end with a review of recent femtosecond X-ray 'snapshot' diffraction from protein nanocrystals, using an X-ray laser which generates pulses so short that they terminate before radiation damage can commence, yet subsequently destroy the sample. In this way, using short pulses instead of freezing, the nexus between dose, resolution and crystal size has been broken, opening the way to time-resolved diffraction without damage for a stream of identical particles.

\section{Introduction}

Textbooks simplify and re-arrange historical events, making them easier to teach and remember; however, this may convey a spurious causality. It is often forgotten, for example, that 'Bragg' diffraction was first discovered using polychromatic radiation, at a time when neither the X-ray wavelength nor crystal unit-cell parameters were known. Thus the 'Laue' arrangement, used for the time-resolved diffraction discussed below, was discovered before the more familiar Bragg geometry with monochromatic radiation.

Lawrence Bragg was born in Adelaide in 1890. His father William, as Third Wrangler reading mathematics in Cambridge, had, at the age of 23 , taken up the position of Professor of Mathematics and Physics at the University of South Australia. Alice Springs was named for William's mother-in-law, Alice Todd, wife of the eminent astronomer Charles Todd, who oversaw the installation of the telegraph from Adelaide to Darwin. As a child, Lawrence was thrown off his tricycle by his younger brother, resulting in a damaged left elbow, which affected him for life. His father promptly obtained an X-ray image of the elbow (the first X-ray image in Australia) using a copy of the apparatus described by Roentgen in 1895 [see Roentgen (1923) for an English translation], reporting the discovery of X-rays. Father and son soon became fascinated by Marconi's work and the local wireless telegraphy installations in the early years of the new century, when the father also published on the absorption of alpha particles and electrons, for which he was elected a Fellow of The Royal Society. These were the days of the great controversy over whether radiation consisted of particles or waves, and of Einstein's 1905 paper on the photoelectric effect, father William favoring the particle model for X-rays, influenced in part by the particle tracks in Wilson's cloud chamber.

The family moved to Leeds in 1908. Lawrence read mathematics at Cambridge, in which he obtained first-class honors, changing then to physics. His lecturers included C. T. R. Wilson, J. J. Thomson and J. H. Jeans, and included informal discussions with Bohr. The general view in Trinity was that 'Braggs were good at experiments, Thomsons were not'. But it was Sommerfeld, in correspondence with William, who stressed the importance of a diffraction experiment to resolve the wave-particle controversy for X-rays. The result in Sommerfeld's Institute in Munich, at the suggestion of von Laue, and supported by Ewald's theory, was the famous experiment by Sommerfeld's experimental assistant Friedrich, and publication of the Friedrich and Knipping paper reporting sharp 'Bragg' spots from ZnS crystals irradiated with polychromatic X-rays in August 1912 (Friedrich et al., 1912). Laue interpreted these patterns as due to characteristic inner-shell radiation from the atoms of the crystal at several wavelengths, diffracted by the surrounding lattice, and assumed (incorrectly) a simple cubic lattice of $\mathrm{ZnS}$ molecules. Lawrence's rapidly following paper, in November 1912 (Bragg, 1912) drew on ideas from the local mineralogists and crystallographers Gossling, Pope and Barlow to assume a face-centered structure, but a continuous range of incident wavelengths to interpret Friedrich and Knipping's results. In modern terms we see that the 'thick' Ewald sphere resulting from polychromatic illumination embedded in a sharp three-dimensional reciprocal lattice produces sharp diffracted beams; however, this was not clear at the time, since full three-dimensional intensity calculations would await the work of Ewald and Darwin. The 
broad radiation spectrum had greatly facilitated Knipping's accidental discovery (after many failures, he surrounded the irradiated crystal with photographic plates), avoiding the need for a goniometer. Lawrence imagined the crystal planes responsible for a row of systematic reflections to act like a stack of half-silvered mirrors, of spacing $d$ (hence 'reflexions'), lying parallel to the cleavage planes of the crystal, which expressed its symmetries. He said he could remember the exact location in the college Backs where it suddenly dawned on him that the spots were due to reflection by (e.g. horizontal) planes, and confirmed this by noting changes in the shape of the spots with increasing camera length $L$ (they were focused in the downward direction across the plate, but not across it, because of the vertical displacement of the reflecting crystal planes within the crystal). This was the key insight which allowed him to use the formula for interference of light from a thin liquid film as the basis for 'Bragg's law', a specular but wavelength-selective condition for given scattering angle. Lawrence then imagined the crystal to be 'manufacturing light of definite wavelengths' equal to the path difference divided by the number $n$ of wavelengths, namely $2 d \sin \theta / n$ ( $2 \theta$ is the scattering angle). He was able to account for all the experimental spots using this scheme (extended to three dimensions). In Leeds, his father pursued the use of Bragg diffraction for the purposes of developing an X-ray spectrometer, and in 1913 analysed Barkla's inner-shell emission lines. (This later allowed Moseley to determine the atomic number of the elements.) Further progress required accurate measurement of the X-ray wavelength in order to determine cell constants - this was achieved by father William in 1913 (Bragg, 1913) using Avogadro's number, the known sample density and assumed lattice. In later work, Lawrence's experiments used the reflection geometry and monochromatic X-rays provided by a crystal monochromator to find atom positions in planes parallel to cleavage planes by reflection. This is the 'Bragg geometry' - it is interesting to see how modern usage now refers to the original transmission geometry, and the use of polychromatic radiation, as 'Laue diffraction'. The competing views of the German and British schools were reconciled entirely by Ewald's theory, based on his famous sphere, in 1913, which correctly showed how the crystal can act as its own monochromator. By 1920, the structures of diamond, sodium chloride, zincblende, fluorspar and alumina had been solved, mostly few-parameter structures for which symmetry arguments could be used to address the phase problem. Iron pyrites and calcite were the first structures to be solved which involved undefined atomic position parameters. R. W. James, who had shared a class at Cambridge with Lawrence, returned from Shackelton's 1917 expedition to join Bragg's artillery sound-ranging unit during the war. In 1919, Lawrence accepted a Professorship in Physics at Manchester, taking James with him, to a department already made world famous by Rutherford. There Lawrence led the effort to quantify scattered intensities (using sodium chloride as a model), based on Darwin's 1914 theory, leading to the theory of mosaicity and all its implications. A persistent conceptual difficulty was the need to give up on the idea that all crystals consist of molecules (as might occur in the liquid or gas phase) on lattice sites (as for proteins), rather than a continously bonded network, as the work on diamond or the silicates was suggesting, where the $\mathrm{SiO}_{4}$ tetrahedral building block was discovered (rather than the $\mathrm{SiO}_{3}$ acid molecules expected from solution chemistry). It is remarkable that Lawrence was able to solve a centrosymmetric structure with as many as 14 independent parameters (diopside), working with only slide rule and tables, solving the phase (sign) problem 'by inspection'. This would have involved summing over 17000 terms in Fourier series by hand. As one colleague who worked with him said, 'His great gift was that of a puzzlesolver, who thought visually.'

Having transformed the field of mineralogy by providing an atomistic basis for it, his book on the atomic structure of minerals (1937) marked a turning point in his interests, toward Fourier methods, and his famous work with Williams on shortrange order in alloys. After a time at the National Physical Laboratory, in 1937 he accepted the position as Cavendish Professor of Physics at Cambridge, much to the consternation of the particle-physics community, who certainly did not consider his work 'fundamental', yet which led eventually to the discovery of the structure of DNA and the growth in radio astronomy. About six weeks after his appointment, M. F. Perutz visited him, to show him his first diffraction patterns from haemoglobin, kindling his interest in protein crystallography, which was to remain his chief interest for the rest of his life. During that time, however, he found time for his development of optical diffractometer methods with Lipson, to develop the heavy-atom method (isomorphous replacement) with Perutz, and his two-dimensional bubble models of atomic defects in metals, such as dislocations and grain boundaries. He returned to Adelaide in 1960 for a visit to show his wife his childhood playgrounds along the seashore, but found it much changed. He loved gardening, painting, sailing and literature, somehow found time for a happy family life, and was always said to be a genial, generous and approachable colleague, always enthusiastic, if inclined on occasion toward formality and even pomposity. Essentially a classical physicist, he spent his last years, until his death in 1971, at the Royal Institution, where his public lectures on science were a frequent highlight, due to his enthusiasm and gift for illustration by analogy.

More comprehensive reviews of these events can be found in Thomas \& Phillips (1990), Eckert (2012), Ewald (1962) and the article by Matthews in this issue (Matthews, 2013).

\section{Microdiffraction - X-rays. Spotty rings}

Bragg's work on metals led naturally to the question of how X-ray diffraction might be used to characterize the defect structure of polycrystalline metals by using a fine-focus beam. A full account of this work is given in the article by Kelly in this issue (Kelly, 2013). Bragg therefore encouraged Kellar and Hirsch to build a rotating-anode microdiffraction apparatus (Kellar et al., 1950), which was to be applied to the study of cold-worked metals in order to determine whether line- 
broadening effects were due to strains or to the small subgrain crystal size - it was found that strain was the predominant cause (Hirsch, 1952). The resulting 'spotty ring' patterns also gave information on deformation, recovery and recrystallization. Beam sizes down to $8 \mu \mathrm{m}$ were obtained (almost the same size as used for the X-ray free-electron laser pattern in Fig. 4) for the study of grain sizes down to $0.2 \mu \mathrm{m}$ in beaten gold foils. Microbeam Laue patterns were obtained from Neumann bands a few microns thick in iron, allowing the twin orientations to be determined (Kelly, 1953). Hirsch (1960) provides a comprehensive review of X-ray microdiffraction apparatus at the time, including the optimization of its design, and operating conditions. Pinhole collimation, factors affecting spot size, design of collimators, the use of capillaries, the convergent-beam method and a condition for the observation of 'spotty rings' are all given. (It is interesting to note that these spotty ring patterns could now readily be analysed using the angular correlation functions described below for disentangling orientational disorder among identical randomly oriented particles in solution.)

X-ray microdiffraction methods have advanced rapidly since that time (for a review, see Ice et al., 2011). Beam diameters as small as $7 \mathrm{~nm}$ diameter have been formed using Kirkpatrick-Baez optics and deformable mirrors at $20 \mathrm{kV}$ on synchrotrons (Mimura et al., 2010), and it is reasonable to expect $1 \mathrm{~nm}$ hard X-ray beams before long. In development of the work of Hirsch, Kelly, Gay and others on microdiffraction from deformed metals, for example, Levine et al. (2012) have recently studied the dislocation cell structure from a single dislocation cell wall using depth-resolved scattering from a $500 \mathrm{~nm}$ diameter beam at $14 \mathrm{kV}$, giving line profiles from the 006 reflection in deformed $\mathrm{Cu}$. This work resolves long-standing controversies in this field over the manner in which (non-unique) profiles from the many cell walls, which normally contribute, are summed.

\section{Microdiffraction - electrons and scanning transmission electron microscopy (STEM)}

Bragg had supported the development of electron microscopy through his recruitment of V. E. Cosslett in 1946, leading to decades of exciting developments at the Cavendish, and in the Oxford Materials department, following the move of P. B. Hirsch to Oxford in 1966. Cosslett himself later developed the X-ray shadow-image microscope, using an electron gun focused to a microspot (whose sized fixed the X-ray resolution) on an X-ray target (Cosslett \& Nixon, 1960). This arrangment, using a field-emission scanning electron microscopy (SEM) gun which allows scanning of the spot over a thin-film vacuum window X-ray target, has been the subject of much development since, and is now a commercial product offered by at least three companies. Here was a field where the formation of submicron beams using magnetic lenses was routine. It began when Mollenstedt, in 1937, was asked by W. Kossel in Danzig to build a $45 \mathrm{kV}$ convergent-beam electron diffraction (CBED) camera (Mollenstedt, 1989). The idea was suggested by the earlier observation of X-ray Kossel patterns and electron Kikuchi patterns in 1928. These patterns may be thought of as due to internal sources of radiation from atomic sites in the crystal, just as Sommerfeld and Laue had first pictured Bragg diffraction. Mollenstedt's CBED camera would supply an external source of diverging radiation. His design (for his diploma thesis project) used a plasma discharge in a wine bottle as the electron source. The vacuum at the specimen was $10^{-3}$ Torr, and the probe size about $40 \mu \mathrm{m}$. With such a large probe, contamination was not a problem, despite the poor vacuum. Using flakes of mica as a sample, CBED patterns were obtained not greatly different from the patterns obtainable on the most modern electron microscopes. Deviations from the kinematic theory were immediately noted. Improved $65 \mathrm{kV}$ and $750 \mathrm{kV}$ machines were also built, before the war brought developments to an end. It was soon realised that the large convergence angle needed to form a small probe could provide useful information within crystal rocking curves, taken from regions smaller than one mosaic block (MacGillavry, 1940). This led to the development of the CBED method and its application to problems of space-group determination for nanocrystals (Shmueli et al., 2013), where the multiple scattering of fast electrons renders the patterns immediately sensitive to the absence of inversion symmetry, unlike X-ray diffraction. Quantitative analysis of low-order CBED rocking curves, made possible by fast digital solution of multiple scattering equations, can also provide accurate maps of the chemical bonds between atoms in inorganic crystals, as shown in Fig. 1 for the copper-oxide bond in cuprite (Zuo et al., 1999). The figure makes an instructive comparison with Bragg's first two-dimensional density map (of diopside) in his development of Fourier methods in crystallography (Bragg, 1929), an idea he attributes to his father. This lead to the analysis of chemical bonds by X-ray diffraction - in hindsight we can now see that problems with extinction errors in that approach are better avoided using the electron diffraction CBED method. The CBED technique depends on the greatly increased sensitivity of low-order electron structure factors

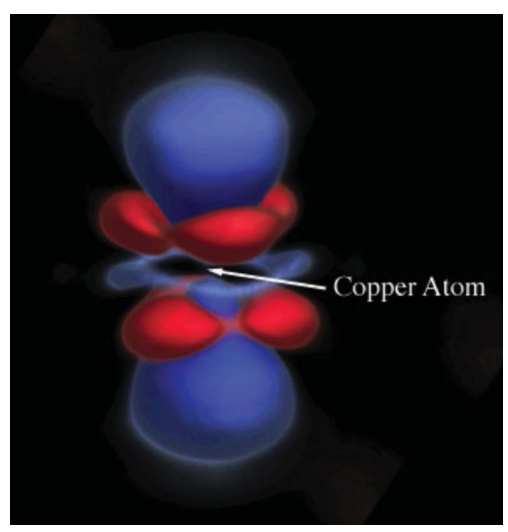

\section{Figure 1}

Experimentally measured charge-density difference map for the copperoxide bond in cuprite. Obtained by quantitative comparison of elastic energy-filtered CBED patterns with multiple scattering simulations. The density is calibrated in electron units per unit volume, and so from spherical harmonic fitting gives separate contributions of ionic and covalent bond charge. 


\section{Bragg centennial}

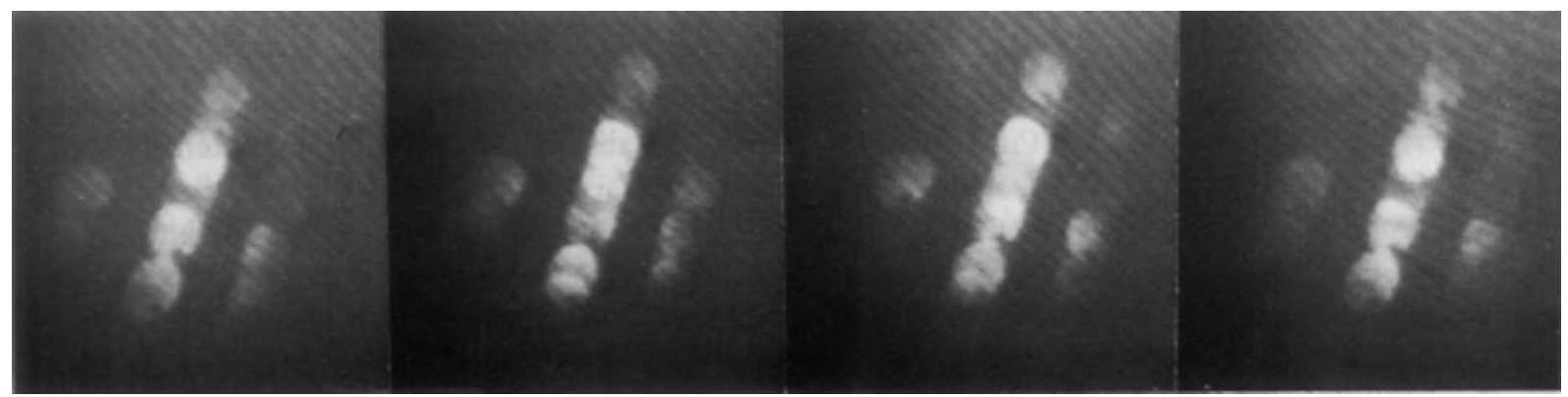

Figure 2

Transmission electron microdiffraction patterns from a mineral using STEM to give a $0.3 \mathrm{~nm}$ probe, smaller than the unit cell. The pattern is seen to repeat as the probe is scanned across a single unit cell, from left to right. Reproduced with permission from Cowley (1981). Copyright (1981) Elsevier.

relative to X-ray ones, allowing extinction-free measurements to be made from regions smaller than one mosaic block, and based on the perfect crystal theory. CBED patterns also allow rather accurate structure-factor phase measurement, while the high-order Laue zone (HOLZ) lines provide a method of strain mapping at the nanometer scale. As one example among many, strain maps have been obtained in this way within a single transistor with $37 \mathrm{~nm}$ gate length using a beam diameter of about $6 \mathrm{~nm}$ to measure strains down to about $10^{-4}$ (Zhang et al., 2006). Here the effects of multiple elastic scattering cause deviations from Bragg's law, giving maxima or minima in HOLZ line positions away from the Bragg condition, so that matching of the entire CBED scattered intensity distribution with simulations is needed for greatest accuracy [for a review of electron microdiffraction with full references, see Spence \& Zuo (1992)].

Probe sizes shrank greatly to sub-nanometer dimensions with the development of the cold field-emission electron gun and the scanning transmission electron microscope by A. Crewe (Crewe et al., 1970). The ability to obtain atomic resolution images and energy-loss spectra from the same region that produced the microdiffraction pattern has since added immensely to the power of this method, which offers a form of absorption spectroscopy in the soft-X-ray energy range with unparalleled spatial resolution. Fig. 2 shows a remarkable microdiffraction pattern recorded using STEM with a $0.3 \mathrm{~nm}$ diameter electron probe, whose diameter is smaller than one unit cell of the crystalline transmission sample (Cowley, 1981). The pattern is seen to repeat as the probe is displaced from one side of the unit cell to the other by one lattice vector. A series of papers has developed the use of this approach for the study of atomic defects in condensedmatter physics (Zhu \& Cowley, 1982; Cowley \& Spence, 1981; Spence \& Zuo, 1992). Fig. 3 shows an enlarged view of a similar pattern recorded with the coherent electron probe stationary at one point within the unit cell of $\mathrm{FeS}_{2}$ (Terauchi et al., 1994), showing interference between overlapping orders. It is instructive to see how Bragg's law operates when the probe becomes smaller (narrower) than one unit cell of the crystal. If aberrations are neglected, so that we can assume a diffractionlimited probe, one may combine Rayleigh's resolution criteria (treating the focused electron beam as the image of a point source) with Bragg's law to relate the probe size to a condition for overlap of orders. If the full width at half maximum for the probe is $w$, then an illumination semiangle of $\theta_{\mathrm{R}}=0.61 \lambda / w$ is required. But Bragg's law for small angles is $\theta_{\mathrm{B}}=0.5 \lambda / a$, where $a$ is the lattice period, so we see that the beam width $w$ becomes equal to the lateral period of the crystal $a$ when the coherent orders overlap by about $20 \%$. For larger overlaps, it becomes impossible to find the centers of the discs to define the reciprocal lattice, and in this sense Bragg's law 'fails' once the beam becomes much smaller than the crystal unit cell. Under those conditions the scattering pattern intensity distribution depends on the location of the probe within the cell. Then the symmetry of the pattern, rather than reflecting the space-group of the sample, gives the local point group reckoned about the center of the probe position in the cell. These effects are the basis of dark-field crystal imaging in STEM (Spence \& Cowley, 1978) and of the ptychography method for crystals (Rodenburg, 2008). It is

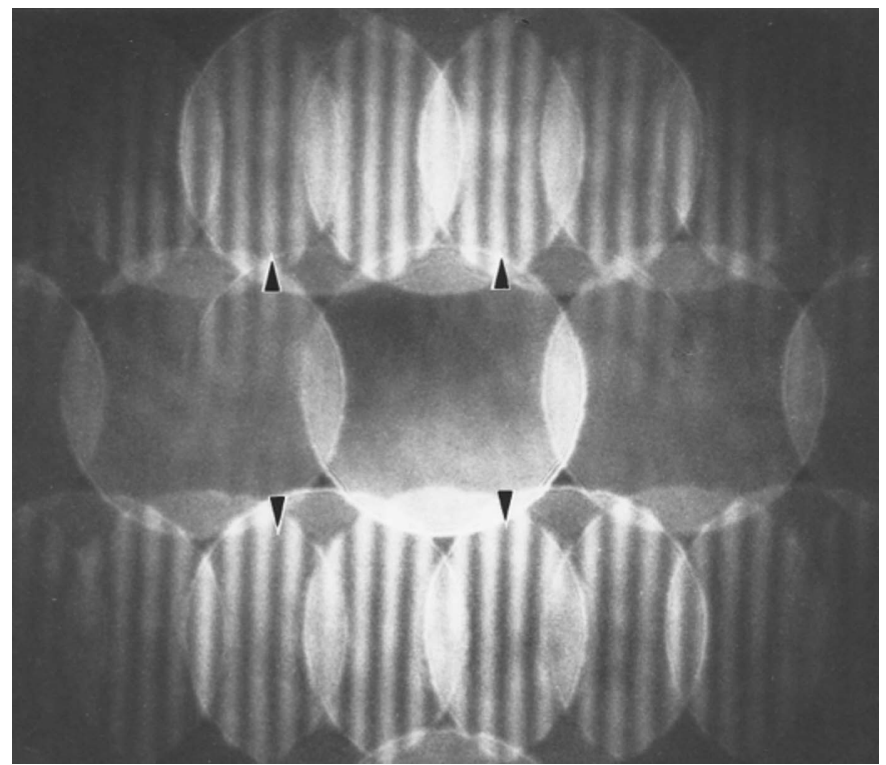

Figure 3

Coherent CBED pattern from $\mathrm{FeS}_{2}$ with the beam along [100] $\left(d_{001}=\right.$ $0.54 \mathrm{~nm}, 200 \mathrm{kV})$, showing interference between overlapping Bragg beams. The Bragg disc directly below the central disc is (020). Arrows indicate contrast reversals due to structure-factor phases. Reproduced with permission from Terauchi et al. (1994). Copyright (1994) Elsevier. 
readily shown that even-order aberrations cancel on achromatic circles passing through the midpoints between Bragg reflections.

The formation of such small probes requires a high degree of spatial coherence. Bragg was well aware of the possibilities offered by coherent sources from an early stage - he attributed his interest in optics to his early lectures from C. T. R. Wilson. His paper A new type of 'X-ray Microscope' in 1939 (Bragg, 1939) described the use of an optical bench for reconstruction of projected crystal density maps by illuminating pinholes, on a two-dimensional lattice in a mask, with coherent light, obtained from a pinhole in front of a mercury lamp. The size of the pinhole was proportional to the measured X-ray structure factors, and phase plates across the pinholes were use to apply phase shifts. Gabor's later paper (Gabor, 1948), proposing electron holography as a method of aberration correction, used the microdiffraction geometry with a coherent diverging beam, and was followed by a paper by Bragg which relates in-line holography to the heavy-atom method of protein crystallography, and discusses the twinimage problem in novel terms (Bragg, 1950). With the development of the laser for many experiments in Fourier optics, it was natural to attempt similar things with coherent electrons and X-ray beams, a process which continues to this day [highresolution X-ray holography using an X-ray laser is demonstrated in Marchesini et al. (2008)]. Spatial coherence requires a point source (such as an electron-field emitter or an X-ray laser), which, if focused upstream of a sample by a defocus distance $\Delta$, also defines the geometry of microdiffraction. This geometry, which, for a mask-like object, produces a shadow image magnified by $L / \Delta$ ( $L$ is the camera length between sample and detector) together with Fresnel edge fringes, has proven extremely rich, giving us a great array of phenomena which are still under investigation (Spence \& Zuo, 1992). These include:

(i) In-line holography, for partially transparent samples, as first attempted using electrons by Haine \& Mulvey (1952), and by Lin \& Cowley (1986) and others using STEM.

(ii) The phenomena of Talbot self-imaging of periodic objects, with magnification, which has now been demonstrated using both electron and X-ray radiation (Cowley, 1998).

(iii) The Tanaka wide-angle CBED method, in which overlap of adjacent CBED orders is prevented to allow symmetry determination in large-unit-cell crystals (Morniroli, 2002).

(iv) The electron Ronchigrams, which are used to align modern aberration-corrected STEM instruments and measure aberration coefficients (Cowley, 1998; Lupini, 2011). It may be shown, for example, that the local magnification of the Ronchigram shadow image is proportional to the second derivative of the wave-aberration function. Fourier transforms of small patches of a Ronchigram give the Thon diffractograms used for focusing.

(v) The ptychography method, first proposed for superresolution imaging of thin crystals, but recently extended to non-periodic samples using electrons, visible light and X-ray beams (Rodenburg, 2008). (vi) The low-voltage point-projection field emission microscope (Weierstall et al., 1999). It has recently been shown that by using a pulsed laser to produce photofield emission from a $\mathrm{GaN}$ tip, this lensless, diverging-beam arrangement minimizes Coulomb interactions, and so is ideal for fast imaging (Spence et al., 2010). The resolution is approximately equal to the virtual source size within the cold field emission tip, but sample penetration is very limited. For molecules on conductive graphene, useful images might be obtained, but hydrated molecules cannot be studied. The question of radiation damage at the very low beam energies involved (e.g. $40 \mathrm{eV}$ ) has been extensively discussed [see Spence et al. (2010) for references].

(vii) The dark-field (not high-angle dark field) incoherent imaging mode in STEM, which uses microdiffraction patterns such as that shown in Fig. 3, obtained from sub-nanometer regions, to form a transmission image from the low-angle elastic scattering as the probe is scanned across the sample (Spence \& Cowley, 1978).

\section{Fast microdiffraction. X-ray lasers}

In Lawrence's pre-sychrotron time, time-resolved X-ray diffraction was hardly a possibility. While much useful research has been done using conventional synchrotrons even at picosecond timescales, it has been the invention of the pulsed free-electron X-ray laser (XFEL) which has recently transformed this field. Microdiffraction using brief pulses suggests the possibility of making movies of atomic and molecular processes, if the phase problem can be solved, and, for high resolution, if sufficient high-angle scattering can accumulated. If an atomic process can be found which may be triggered repeatedly by a fast optical pulse (such as photosynthesis or plasmon excitation), the photocathode of an electron microscope, diffraction camera or X-ray laser can then be excited after a time delay $\Delta T$, to provide one two-dimensional snapshot frame of the excited state of the sample. The process must be repeated, with the sample in a range of orientations, to provide a threedimensional reconstruction, and again with a different delay for each frame of a movie. Many issues arise, notably the effects of radiation damage for the high fluences needed per shot, the question of whether a crystalline or single-particle sample can be used, and the question of whether all this is best achieved using electron beams or X-ray beams. Time-resolved protein crystallography (Graber et al., 2010), for example, uses a laser-excited reversible process in a protein crystal involving few-atom motion in a small part of a large molecule within a unit cell which may consist mostly of water. Since an angular integration, normally provided by goniometer control, across the crystal rocking curve is required to obtain structure-factor measurements, and this is not possible during snapshot diffraction, the Laue method with a wide range of beam energies is normally used to provide integrated rather than partial reflections.

Spatial and chromatic coherence is required for highresolution imaging. For electrons, magnetic lenses may be used 


\section{Bragg centennial}

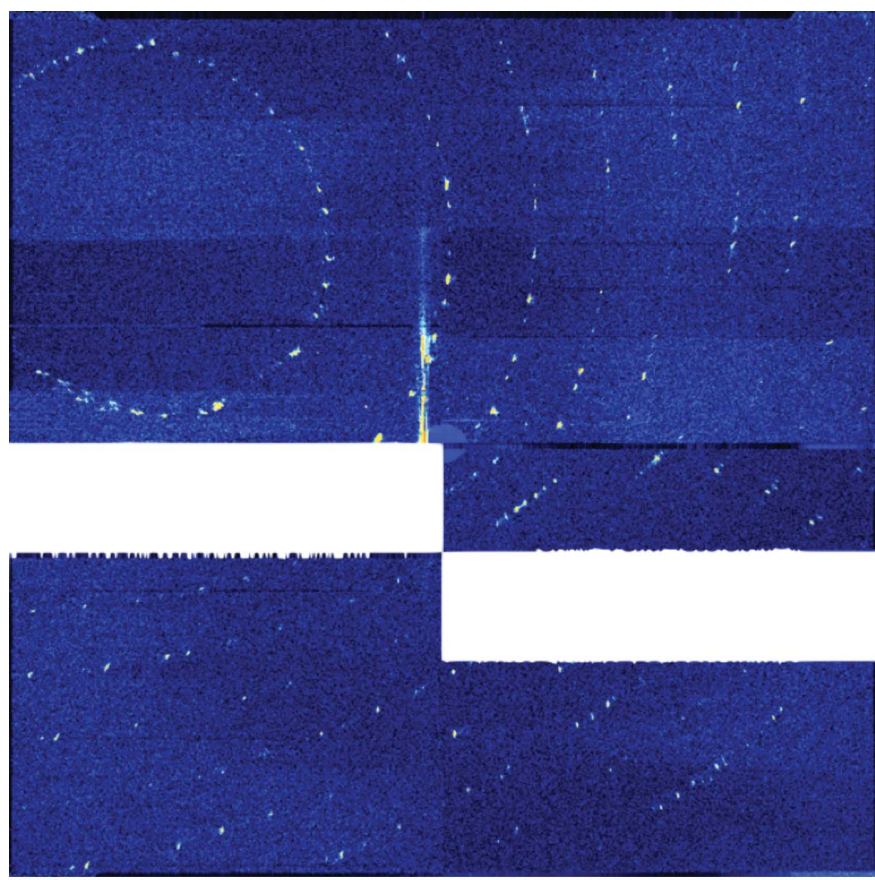

(a)

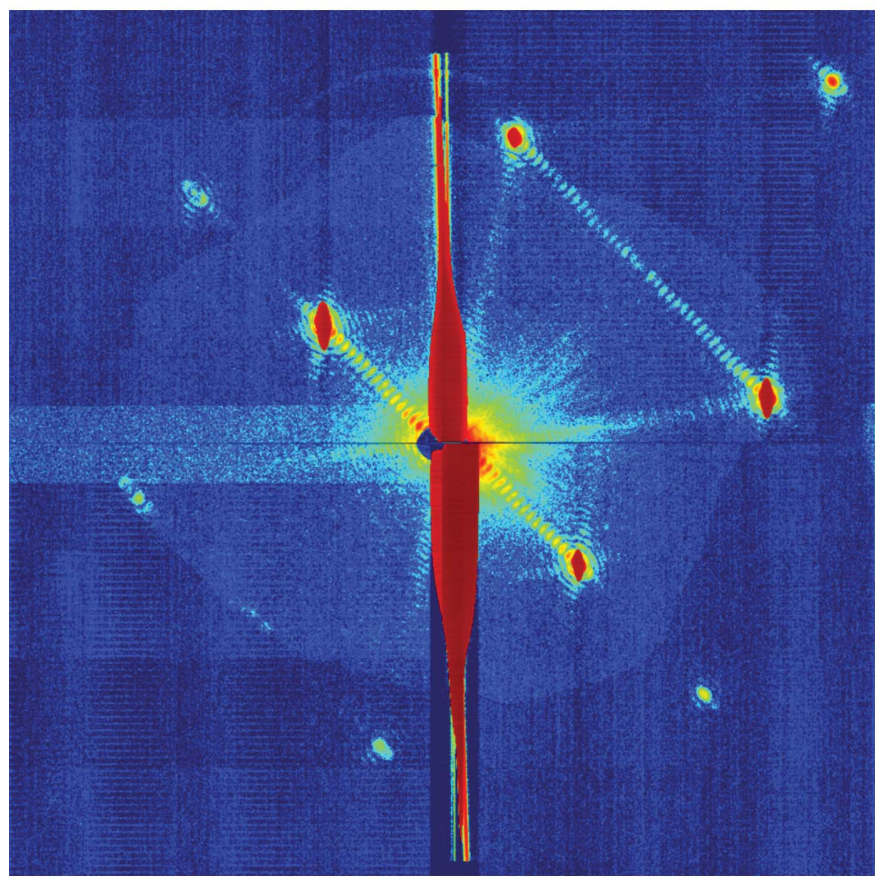

Figure 4

(b)

(a) Snapshot XFEL transmission X-ray diffraction pattern from PS I. This is a single pulse of $70 \mathrm{fs}$ duration, with $2 \mathrm{~mJ}$ of $1.8 \mathrm{kV}$ X-rays at $0.688 \mathrm{~nm}$ wavelength. The resolution is $8.9 \AA$ at the corner. Blank areas are faulty detector panels. The beam diameter was $7 \mu \mathrm{m}$ and this single pulse contained about $10^{12}$ photons. [See Chapman et al. (2011) for details.] (b) Recorded under similar conditions to $(a)$, but using a smaller camera length for higher angular resolution of low orders. This snapshot pattern from one protein nanocrystal shows the Fourier transform of the needleshaped nanocrystals laid down around reciprocal-lattice sites. The number of subsidiary maxima between Bragg reflections shows that there are 19 periods of the crystal running between facets along the diagonal direction of the fine fringes. The vertical streak is diffraction from the column of liquid water in which the nanocrystals run [see Spence et al. (2012) for details]. to form a single-shot image directly; however, except at very high beam energies (or when using one electron per shot), the Coulomb interaction between electrons at crossovers in lenses limits the speed of the imaging, by introducing unwanted beam divergence, energy spread and coherence, with resulting loss of phase contrast. Valuable results have, however, been obtained in the application of this method to materials problems (Reed et al., 2009). One solution is repeated excitation, with stroboscopic recording at high (e.g. MHz) repetition rates; however, exactly reproducible atomic processes are difficult to find, so that imaging of electronic excitations has been more successful (Barwick et al., 2009). Fast electron diffraction methods (Sciaini \& Miller, 2011), using short apparatus, with few, optimized lenses, radio-frequency (RF) pulse compression and novel electron sources may soon be competitive with self-amplified spontaneous emission (SASE) mode X-ray lasers. For a recent 'table-top' $3 \mathrm{MeV}$ electron diffraction system, for example, it was possible to generate $10^{6}$ electrons in a $100 \mathrm{fs}$ pulse with a $500 \mu \mathrm{m}$ diameter beam, with small values of beam divergence and energy spread producing sharp Bragg peaks in transmission (Muro'oka et al., 2011). The much larger elastic cross section of fast electrons compared with X-rays makes up for much of the reduction in incident pulse fluence, and inelastic scattering cross sections are relatively smaller.

It has recently been discovered that radiation damage may be avoided through the use of sufficiently brief pulses (of perhaps $70 \mathrm{fs}$ duration), which terminate before radiation damage begins (Chapman et al., 2011). Radiation damage destroys fine detail in a sample first, as demonstrated by Bragg spot-fading experiments. But by packing enough photons or electrons into a femtosecond pulse, one may obtain an almost unlimited amount of the near-instantaneous elastic scattering before the development of inelastic processes (such as a photoelectron cascade) vaporize the sample. This is shown in Fig. 4(a) for the case of a photosystem I (PS I) protein nanocrystal, using the arrangement shown in Fig. 5. This pattern was produced by a single $1.8 \mathrm{kV}$ X-ray pulse of $70 \mathrm{fs}$ duration, containing about $10^{12}$ photons. The pulses arrive with a repetition rate of perhaps $100 \mathrm{~Hz}$, and diffraction patterns are recorded and read out at this rate, producing many terrabytes of data. [More recent work has extended these results to atomic resolution (Boutet et al., 2012).] In the past, several papers have addressed the question 'What is the smallest protein crystal which, subject to the limits of radiation damage, can provide data of a given resolution?' (see e.g. Glaeser, 1999). Our new 'diffract-and-destroy' approach now breaks this nexus between dose, resolution, damage and crystal size. If a stream of identical samples (such as hydrated bioparticles in the same conformation) lying in random orientations can be supplied, running in single file across the pulsed beam, and their orientations determined (so that the data may be merged into a three-dimensional diffraction volume), then the diffraction data for a three-dimensional image reconstruction may be obtained. Iterative methods may then be used to solve the phase problem [see Spence (2006) for a review of this field of 'lensless imaging']. 


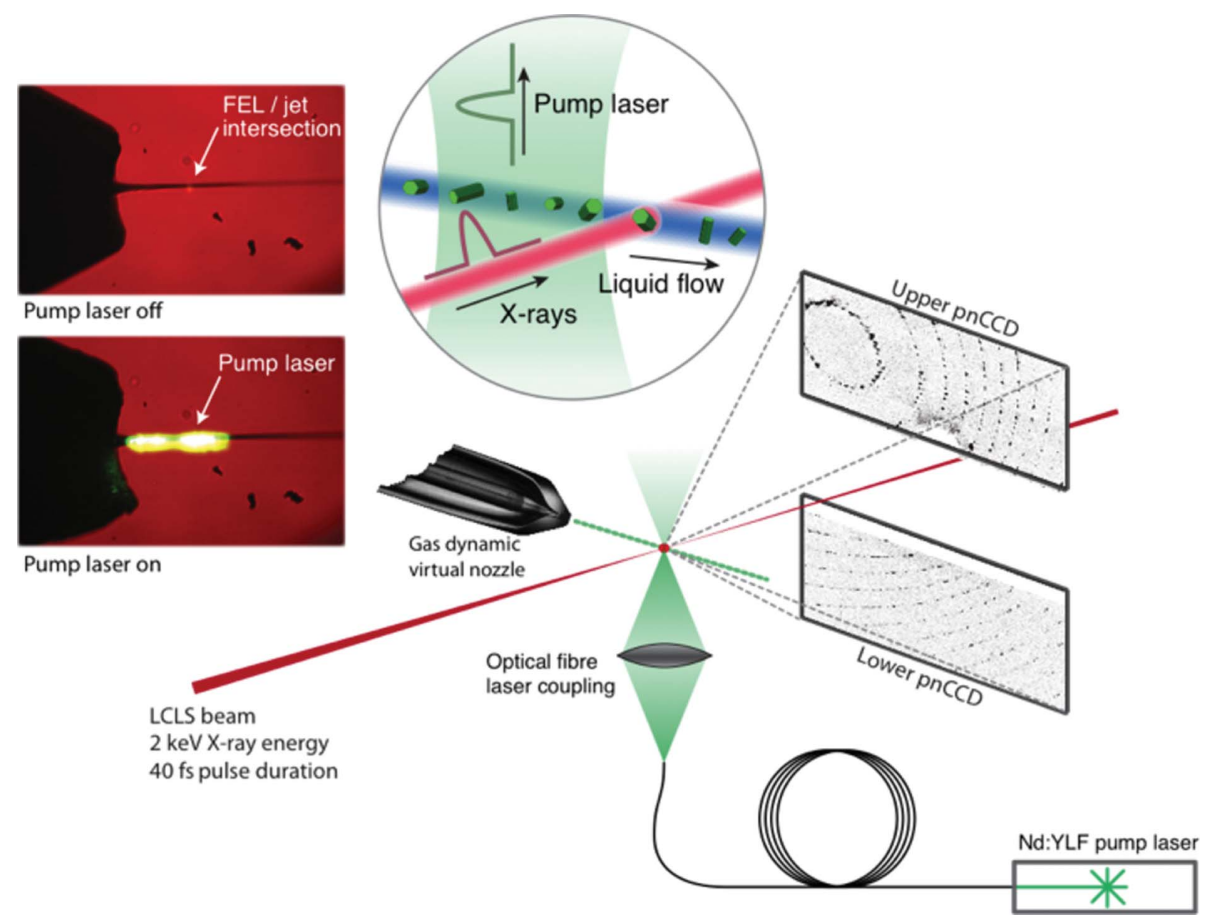

Figure 5

Experimental arrangement used for fast time-resolved microdiffraction using a pulsed X-ray laser. The gas dynamic virtual nozzle provides a hydrated stream of bioparticles running in single file. The inset shows a pump laser exciting fluorescence on molecules of PS I as for sunlight falling on a leaf. (From Aquila et al., 2012.)

Over the past decade we have developed both the liquid-jet hydrated particle delivery device for these experiments (Weierstall et al., 2012) and the Monte Carlo method of diffraction data analysis for protein nanocrystals (Kirian et al., 2011; White et al., 2012). Since a wide range of energies cannot be provided by the SASE mode XFELs, we rely on the occurence of nanocrystals arriving with a uniform distribution of orientations, by chance, to provide the rocking-curve integration. For this fast X-ray microdiffraction method of 'serial crystallography' we use a beam diameter of about $1 \mu \mathrm{m}$, with protein nanocrystals and a gas-focused Rayleigh single-file liquid droplet beam of about the same diameter.

Snapshot patterns recorded on a low-angle detector at high angular resolution also show additional 'shape-transform' fine structure, which may be used to determine the number of unit cells in the nanocrystals, as shown in Fig. 4(b). They also assist in solving the phase problem by the oversampling method here we sum the data from nanocrystals of many different sizes and 'divide out' the particle-size distribution before applying iterative phasing methods, as described in Spence $e t$ al. (2011). Auto-indexing of tens of thousands of patterns allows them to be merged in the correct relative orientation.

While the femtosecond X-ray pulses are used to avoid radiation damage, pump-probe experiments in the jet can also be executed at the slower microsecond timescales of protein interactions. Early results of these experiments (Aquila et al., 2012) show clear differences between the measured structure factors (obtained by merging partial reflections from hundreds of thousands of nanocrystals) from the ground state of photosystem I-ferredoxin cocrystals, and the structures formed 5 and $10 \mu$ s after flash illumination by green light at $0.8 \mathrm{~nm}$ resolution. This is expected, as the small ferredoxin molecule undocks from the larger PS I molecule as a result of a series of electron-transfer oxidation reactions, in response to light. By developing this 'pump-probe-in-a-jet' method to higher resolution, we hope soon to image the molecular processes responsible for oxygen production and water splitting in all green plants, under the action of sunlight, that maintain our biosphere.

Similar fast microdiffraction snapshot X-ray imaging has been achieved from individual Mimiviruses, and reconstructed at about $30 \mathrm{~nm}$ resolution in two dimensions (Seibert et al., 2011). A single very large virus such as this scatters only about a million photons (with $10^{12}$ incident), and less than one photon per shot at the high angles needed for $1 \mathrm{~nm}$ resolution. It is readily shown that soft $\mathrm{X}$-ray scattering from a sphere falls off as the inverse fourth power of the scattering angle, a severe penalty for high-resolution single-particle imaging using $\mathrm{X}$-rays. As for cryo-electron microscopy, some advantage may be gained using helical or icosohedral particles with internal symmetry. The paper by Seibert et al. reports the first X-ray diffraction pattern from a single particle (a virus), and uses the microdiffraction method. The orientation-determination problem here with continuous scattering is more difficult, but appears solvable using both expectation maximization and Hilbert space methods. The phase problem for these singleparticle snapshot diffraction patterns is also solved using iterative methods, and may be integrated with the orientation and conformation sorting routines [see Spence et al. (2012) for a review]. 
A final remarkable development has been the emergence of $a b$ initio methods for reconstructing an image of one particle using the scattering from many copies, in random orientations, without modeling. This method, which may be applied to identical particles frozen in either space or time, is based on angular correlation functions, has now been demonstrated experimentally for inorganic samples (Saldin et al., 2011), and is in the process of being applied to the snapshot imaging of proteins in solution which are undergoing chemical reactions.

\section{Summary}

Microdiffraction techniques using X-rays and electrons have advanced to lengths unthinkable in Bragg's day. Electron beams narrower than one atom can now be produced using aberration-corrected STEM instruments, and few-nanometerwide coherent X-ray beams have already been produced (Mimura et al., 2010). Bragg appreciated the importance of this, and understood that electron microscopy could provide a vital contribution.

Bragg diffraction has been the basis of more Nobel awards than any other effect in science. His encapsulation, using ideas from optics, of the essence of the much more complex theories of Darwin and Ewald (which predicted both intensities and directions of Bragg beams) arose from attention to subtle detail (observation of the shape of the spots as the detector distance was changed), his background in optics (rather than Sommerfeld's preoccupation with the quantum theory of Bremsstrahlung), a fortunate association with local mineralogists and of course his (later difficult) relationship with his father. Bragg the younger once wrote that 'The fatal enemy of research is a full engagement book ... the muse of science is capricious, but flees from the busy man' and so would perhaps have agreed with Seneca that 'a busy life is a wasted life'. No doubt he would have scoffed at the idea that email could assist research. He was a keen bird watcher - on his departure for the Royal Institution, the Cavendish presented him with a pair of field glasses. He was a fine exemplar of that idea of the ancient Greeks, that the only thing needed for a happy life is a project one is enthusiastic about.

This work was supported by DOE award DE-FG0302ER45996. I am most grateful to Professor Sir Peter Hirsch for useful discussions.

\section{References}

Aquila, S., et al. (2012). Opt. Express, 20, 2707-2709.

Barwick, B., Flannigan, D. J. \& Zewail, A. H. (2009). Nature (London), 462, 902-906.

Boutet, S., et al. (2012). Science, 337, 362-364.

Bragg, W. H. (1913). Proc R. Soc. Ser. A, 89, 246-248.

Bragg, W. L. (1912). Proc. Cambridge Philos. Soc. 17, $43-57$.

Bragg, W. L. (1929). Proc R. Soc. Ser. A, 123, 527-529.

Bragg, W. L. (1939). Nature (London), 143, 678-680.

Bragg, W. L. (1950). Nature (London), 166, 399-402.

Chapman, H. N., et al. (2011). Nature (London), 470, 73-75.

Cosslett, V. E. \& Nixon, W. C. (1960). X-ray Microscopy. Cambridge

University Press.

Cowley, J. M. (1981). Ultramicroscopy, 7, 19-23.
Cowley, J. M. (1998). Diffraction Physics, 3rd ed. New York: North Holland.

Cowley, J. M. \& Spence, J. C. H. (1981). Ultramicroscopy, 6, 359365.

Crewe, A., Wall, J. \& Langmore, J. (1970). Science, 168, 1338-1340.

Eckert, M. (2012). Acta Cryst. A68, 30-39.

Ewald, P. P. (1962). Editor. Fifty Years of X-ray Diffraction. Utrecht: NVA Oosthoek's Uitgeversmaatschappij.

Friedrich, W., Knipping, P. \& Laue, M. (1912). Sitzungsber. K. Bayer. Akad. Wiss., pp. 303-322.

Gabor, D. (1948). Nature (London), 161, 777-778.

Glaeser, R. M. (1999). J. Struct. Biol. 128, 3-14.

Graber, T., et al. (2010). J. Synchrotron Rad. 18, 658-670.

Haine, M. E. \& Mulvey, T. (1952). J. Opt. Soc. Am. 42, 763-766.

Hirsch, P. B. (1952). Acta Cryst. 5, 172-175.

Hirsch, P. B. (1960). X-ray Diffraction by Polycrystalline Materials, edited by H. S. Peiser, H. P. Rooksby \& A. J. C. Wilson, ch. 9. London: Institute of Physics/Chapman and Hall.

Ice, G. E., Budai, J. D. \& Pang, J. W. L. (2011). Science, 334, 12341239.

Kellar, J. N., Hirsch, P. B. \& Thorp, J. S. (1950). Nature (London), 165, 554-557.

Kelly, A. (1953). Proc. Phys. Soc. London. Ser. A, 66, 403-409.

Kelly, A. (2013). Acta Cryst. A69, 16-24.

Kirian, R. A., White, T. A., Holton, J. M., Chapman, H. N., Fromme, P., Barty, A., Lomb, L., Aquila, A., Maia, F. R. N. C., Martin, A. V., Fromme, R., Wang, X., Hunter, M. S., Schmidt, K. E. \& Spence, J. C. H. (2011). Acta Cryst. A67, 131-140.

Levine, L. E., Geantil, P., Larson, B. C., Tischler, J. Z., Kassner, M. E. \& Liu, W. (2012). J. Appl. Cryst. 45, 157-165.

Lin, J. A. \& Cowley, J. M. (1986). Ultramicroscopy, 19, 179-182.

Lupini, A. (2011). Scanning Transmission Electron Microscopy, edited by S. J. Pennycook \& P. D. Nellist. New York: Springer.

MacGillavry, C. H. (1940). Physica, 7, 329-332.

Marchesini, S., et al. (2008). Nat. Photonics, 2, 560-563.

Matthews, B. W. (2013). Acta Cryst. A69, 34-36.

Mimura, H. et al. (2010). Nat. Phys. 6, 122-125.

Mollenstedt, G. (1989). Phys Status Solidi A, 116, 13-16.

Morniroli, J.-P. (2002). Large-Angle Convergent-Beam Electron Diffraction. Paris: Society of French Microscopists.

Muro'oka, Y., Naruse, N., Sakakihara, S., Ishimaru, M., Yang, J. \& Tanimura, K. (2011). Appl. Phys. Lett. 98, 251903.

Reed, B. W., Armstrong, M. R., Browning, N. D., Campbell, G. H., Evans, J. E., Lagrange, T. \& Masiel, D. J. (2009). Microsc. Microanal. 15, 272-281.

Rodenburg, J. M. (2008). Advances in Imaging and Electron Physics, Vol. 150, edited by P. W. Hawkes, pp. 87-184. New York: Elsevier.

Roentgen, W. (1923). Am. J. Roentgenol. Radium Ther. 10, 320326.

Saldin, D. K., Poon, H. C., Bogan, M. J., Marchesini, S., Shapiro, D. A., Kirian, R. A., Weierstall, U. \& Spence, J. C. H. (2011). Phys. Rev. Lett. 106, 115501.

Sciaini, G. \& Miller, R. J. D. (2011). Rep. Prog. Phys. 74, 096101.

Seibert, M. M., et al. (2011). Nature (London), 470, 78-81.

Shmueli, U., Flack, H. D. \& Spence, J. C. H. (2013). International Tables for Crystallography, Vol. A, 6th ed., ch. 1.7. In preparation. Spence, J. C. H. (2006). Science of Microscopy, edited by P. Hawkes \& J. C. H. Spence, ch. 18. New York: Springer.

Spence, J. C. H. \& Cowley, J. M. (1978). Optik, 50, 129-138.

Spence, J. C. H., Kirian, R. A., Wang, X., Weierstall, U., Schmidt, K. E., White, T., Barty, A., Chapman, H. N., Marchesini, S. \& Holton, J. (2011). Opt. Express, 19, 2866-2873.

Spence, J. C. H., Vecchione, T. \& Weierstall, U. (2010). Philos. Mag. 90, 4691-4703.

Spence, J. C. H., Weierstall, U. \& Chapman, H. (2012). Rep. Prog. Phys. 75, 102601.

Spence, J. C. H. \& Zuo, J. M. (1992). Electron Microdiffraction. New York: Plenum Press. 


\section{Bragg centennial}

Terauchi, M., Tsuda, K., Kamimura, O., Tanaka, M., Kaneyama, T. \& Honda, T. (1994). Ultramicroscopy, 54, 268-275.

Thomas, J. M. \& Phillips, D. (1990). The Legacy of Sir Lawrence Bragg. Northwood: The Royal Institution.

Weierstall, U., Spence, J. C. H. \& Doak, R. B. (2012). Rev. Sci. Instrum. 83, 035108.

Weierstall, U., Spence, J. C. H., Stevens, M. \& Downing, K. H. (1999). Micron, 30, 335-338.
White, T. A., Kirian, R. A., Martin, A. V., Aquila, A., Nass, K., Barty, A. \& Chapman, H. N. (2012). J. Appl. Cryst. 45, 335-341.

Zhang, P., Kisielowski, C., Istratov, A. A., He, H., Nelson, C., Mardinly, J., Weber, E. \& Spence, J. (2006). Appl. Phys. Lett. 89, 161907.

Zhu, J. \& Cowley, J. M. (1982). Acta Cryst. A38, 718-724.

Zuo, J., Kim, M., O'Keeffe, M. \& Spence, J. (1999). Nature (London), 401, 49-51. 